\title{
UNIVERSAL METHOD OF STOCHASTIC DESCRIPTION OF RANDOM ERROR TERMS OF NAVIGATION MEASURINGS
}

\section{УНИВЕРСАЛЬНЫЙ СПОСОБ СТОХАСТИЧЕСКОГО ОПИСАНИЯ СЛУЧАЙНЫХ ПОГРЕШНОСТЕЙ НАВИГАЦИОННЫХ ИЗМЕРЕНИЙ}

\author{
I.I. Vorohobin, $P h D$, associate professor, V. G. Aleksichin, $P h D$, professor, \\ I.Y. Fusar, PhD student \\ И.И. Ворохобин, к.т.н., доцент, В.Г. Алексишин, к.т.н., профессор, \\ И. Ю. Фусар, аспирант \\ National University «Odessa Maritime Academy», Ukraine \\ Национальный университет «Одесская морская академия», Украина
}

\begin{abstract}
The analysis of statistical these errors of the navigation measuring, got last thirty years in the model supervisions, showed that errors did not submit to the normal law of distributing, and the histograms of their selections contain the surplus number of members in extreme digits, that testifies to the positive excess of law of distributing. Therefore, as alternative to the normal law the models of the mixed laws of distributing of errors were offered, for which characteristic positive excess.

For providing of maximally possible exactness of observations of place of ship it is necessary to know the law of distributing of errors of the navigation measuring. However, at the limited sample size of statistical materials of errors it is not succeeded by standard procedure to define the law of their distributing, although there is possibility to estimate the central moments of distributing. Thus if the histogram of selection has a positive excess, it is possible to use decomposition of closeness of distributing of errors by the orthogonal polynomials Ermit, not having in the order of its analytical expression, and to apply him as closeness of distributing.

It is shown that the variety of laws of distributing of authenticity of random error terms, the feature of which there is the presence of positive excess, can be compatible by the use of orthogonal decomposition with the acquired values of central moments of higher orders. Decomposition of normal closeness of the rationed error in the row of Gramme - Sharle type A by the orthogonal polynomials Ermit is a classic result, however for the standard closeness of law to Gaus of the uncaptioned error in work certain coefficients of row of Gramme - Sharle type A, and led to orthogonal of the Ermit polynomials.

The got result allows offering the universal method of description of closeness of distributing of the uncaptioned casual size by its decomposition in the row of Gramme - Sharle type A, that provides maximal exactness of observation of place of ship at the unknown law of distributing of errors of measuring, that has a positive excess.
\end{abstract}


Keywords: navigation accident rate, laws of distributing of random error terms, orthogonal decomposition of closeness of distributing, the Ermyt's polynomials.

\section{PЕФЕРАТ}

Аналіз статистичних даних похибок навігаційних вимірювань, одержаних останні тридцять років в натурних спостереженнях, показав, що похибки не підкоряються нормальному закону розподілу, а гістограми їх вибірок містять надмірне число членів в крайніх розрядах, що свідчить про позитивний ексцес закону розподілу. Тому як альтернативні нормальному закону були запропоновані моделі змішаних законів розподілу похибок, для яких характерний позитивний ексцес.

Для забезпечення максимально можливої точності обсервацій місця судна необхідно знати закон розподілу похибок навігаційних вимірювань. Проте при обмеженому об'ємі вибірки статистичних матеріалів похибок не вдається за допомогою стандартної процедури визначити закон їх розподілу, хоча $\epsilon$ можливість оцінити центральні моменти розподілу. При цьому якщо гістограма вибірки має позитивний ексцес, то можна використовувати розкладання щільності розподілу похибок за допомогою ортогональних поліномів Ерміту, не маючи в своєму розпорядженні іï аналітичного виразу, і застосувати його як щільність розподілу.

Показано, що різноманітність законів розподілу вірогідності випадкових похибок, особливістю яких є наявність позитивного ексцесу, може бути уніфіковане використанням ортогонального розкладання 3 набутими значеннями центральних моментів вищих порядків. Розкладання нормальної щільності нормованої похибки в ряд Грама - Шарльє типу А за допомогою ортогональних поліномів Ерміту $\epsilon$ класичним результатом, проте для стандартної щільності закону Гаусу ненормованої похибки в роботі визначені коефіцієнти ряду Грама - Шарльє типу А, і доведена ортогональність поліномів Ерміту.

Одержаний результат дозволяє запропонувати універсальний спосіб опису щільності розподілу ненормованої випадкової величини за допомогою іï розкладання в ряд Грама - Шарльє типу А, що забезпечує максимальну точність обсервації місця судна при невідомому законі розподілу похибок вимірювань, що має позитивний ексцес.

Ключові слова: навігаційна аварійність, закони розподілу випадкових похибок, ортогональне розкладання щільності розподілу, поліноми Ерміту.

Біблиографія - 4 джерела, ілюстрацій - немає.

Постановка проблемы в общем виде и ее связь с важными научными или практическими задачами

При дефиците статистических материалов погрешностей навигационных измерений не удается с помощью стандартной процедуры определить закон их распределения, однако можно оценить центральные моменты распределения и если гистограмма выборки имеет «утяжеленные хвосты», то можно 
использовать разложение плотности распределения погрешностей с помощью ортогональных полиномов Эрмита, не располагая ее аналитическим выражением.

Анализ последних достижений и публикаций, в которых начато решение данной проблемы и выделение нерешенных ранее частей общей проблемы

В работе [1] рассмотрена модель формирования смешанных распределений погрешностей навигационных измерений, а в работе [2] предложены два типа смешанных законов распределения погрешностей измерения навигационных параметров. В работе [3] предложено использования обобщенного распределения Пуассона для описания случайных погрешностей.

Формулировка целей статьи (постановка задачи) Целью статьи является разработка универсального способа описания случайных погрешностей навигационных измерений с помощью ортогонального разложения их плотности распределения.

\section{Изложение основного материала исследования с обоснованием полученных научных результатов}

Оценка точности обсервации судна возможна при известном законе распределения векториальной погрешности, аналитический вид плотности которой однозначно определяется выражением законов распределения погрешностей измерений навигационных параметров.

Закон распределения погрешностей навигационных измерений близок к закону Гаусса [1], поэтому можно воспользоваться результатами работы [4], в которой показано, что плотность распределения $f(x)$ центрированной и нормированной случайной величины $x$ можно представить разложением:

$$
f(x)=c_{0} \varphi(x)+c_{1} \varphi^{(1)}(x)+c_{2} \varphi^{(2)}(x) / 2 ! \ldots+c_{i} \varphi^{(i)}(x) / i ! \ldots,
$$

где $\varphi(x)=(2 \pi)^{-1 / 2} \exp \left(-x^{2} / 2\right)$ - нормальная плотность нормированной и центрированной случайной величины.

В работе [4] показано, что производные высших порядков от нормальной плотности $\varphi^{(i)}(x)$ выражаются через ортогональные полиномы Эрмита $H_{i}(x)$ :

$$
\varphi^{(i)}(x)=(-1)^{i} H_{i}(x) \varphi(x),
$$

где $H_{i}(x)=(-1)^{i}\left\{\frac{d^{i}}{d x^{i}}\left[\exp \left(-x^{2} / 2\right)\right]\right\} \exp \left(-x^{2} / 2\right)$.

Коэффициенты $c_{i}$ в формуле (1) вычисляются по формулам:

$$
c_{i}=(2 \pi)^{-1 / 2}(-1)^{i} \int_{-\infty}^{\infty} H_{i}(x) \exp \left(-x^{2} / 2\right) \mathrm{d} x .
$$


В случае, когда случайной величина $x$ является ненормированной, разложение имеет следующий вид:

$$
f(x)=c_{0} \varphi(x)+c_{1} \varphi^{(1)}(x)+c_{2} \varphi^{(2)}(x) / 2 ! . .+c_{i} \varphi^{(i)}(x) / i ! . .
$$

где $\varphi(x)=(2 \pi)^{-1 / 2} \sigma^{-1} \exp \left(-x^{2} / 2 \sigma^{2}\right)$ и $\varphi^{(i)}(x)=(-1)^{i} H_{i}\left(x / \sigma^{2}\right) \varphi(x)$,

причем $\sigma$ - дисперсия исходной плотности $f(x)$.

Для нормированной нормальной плотности ортогональность полиномов Эрмита доказана в работе [3], которая выражается следующим образом:

$$
\int_{-\infty}^{\infty} H_{\mathrm{m}}(\mathrm{x}) H_{\mathrm{n}}(\mathrm{x}) \exp \left(-\mathrm{x}^{2} / 2\right) \mathrm{dx}=\begin{gathered}
0 \quad \text { при } \mathrm{m} \neq \mathrm{n} \\
\mathrm{n} !, \quad \text { при } \mathrm{m}=\mathrm{n}
\end{gathered}
$$

Полиномы Эрмита для ненормированной плотности нормального закона $f(\mathrm{x})=\frac{1}{\sqrt{2 \pi} \sigma} \exp \left(-\mathrm{x}^{2} / 2 \sigma^{2}\right) \quad$ также обладают свойством ортогональности, т. е. удовлетворяют условию:

$$
\int_{-\infty}^{\infty} H_{\mathrm{m}}\left(\mathrm{x} / \sigma^{2}\right) H_{\mathrm{n}}\left(\mathrm{x} / \sigma^{2}\right) \exp \left(-\mathrm{x}^{2} / 2 \sigma^{2}\right) \mathrm{dx}=\begin{gathered}
0 \\
\sigma^{-2 \mathrm{n}} \mathrm{n} !, \quad \text { при } \mathrm{m}=\mathrm{n}
\end{gathered} .
$$

Для поиска выражений коэффициентов $c_{v}$ умножим левую и правую части выражения (2) на $\mathrm{H}_{v}\left(x / \sigma^{2}\right)$, почленно интегрируем и учитываем свойство ортогональности полиномов Эрмита, в итоге получим:

$$
\begin{gathered}
\int_{-\infty}^{\infty} f(x) H_{v}\left(x / \sigma^{2}\right) d x=\frac{c_{0}}{(2 \pi)^{1 / 2} \sigma} \int_{-\infty}^{\infty} H_{0}\left(x / \sigma^{2}\right) H_{v}\left(x / \sigma^{2}\right) \exp \left(-x^{2} / 2 \sigma^{2}\right) d x+ \\
+\ldots+\frac{c_{v}}{(2 \pi)^{1 / 2} \sigma(v !)} \int_{-\infty}^{\infty} H_{v}\left(x / \sigma^{2}\right) H_{v}\left(x / \sigma^{2}\right) \exp \left(-x^{2} / 2 \sigma^{2}\right) d x+\ldots . .
\end{gathered}
$$

Обращаем внимание на то, что все члены полученного ряда (3), кроме члена с коэффициентом $c_{v}$, содержат произведение полиномов Эрмита с разными индексами, это в силу свойства ортогональности полиномов обращает их произведения в ноль. Следовательно:

$$
\int_{-\infty}^{\infty} f(x) H_{v}\left(x / \sigma^{2}\right) d x=\frac{c_{v}}{(2 \pi)^{1 / 2} \sigma(v !)} \int_{-\infty}^{\infty} H_{v}\left(x / \sigma^{2}\right) H_{v}\left(x / \sigma^{2}\right) \exp \left(-x^{2} / 2 \sigma^{2}\right) d x
$$

Используя свойство ортогональности для произведения полиномов Эрмита с одинаковыми индексами, получим:

$$
\int_{-\infty}^{\infty} f(x) H_{v}\left(x / \sigma^{2}\right) d x=\frac{c_{v}}{(2 \pi)^{1 / 2} \sigma(v !)} \sigma^{-2 v} v !(2 \pi)^{1 / 2} \sigma=c_{v} \sigma^{-2 v}
$$


откуда:

$$
c_{v}=\sigma^{2 v} \int_{-\infty}^{\infty} f(x) H_{v}\left(x / \sigma^{2}\right) d x .
$$

Несобственный интеграл в правой части равенства является многочленном от моментов плотности распределения $f(x)$. В подинтегральном выражении следует учитывать только четные полиномы Эрмита, так как нечетные содержат переменную $x$ только в нечетных степенях, что определяет их нулевые значения.

Для коэффициентов с четными индексами $c_{2 s}(s=1,2, \ldots)$ справедливо:

$$
\begin{array}{r}
c_{2 s}=\sigma^{4 v} \int_{-\infty}^{\infty} \sum_{i=0}^{v}(-1)^{i} \sigma^{-2(2 s-i)} a_{i v} x^{2(v-i)} f(x) d x= \\
\sum_{i=0}^{v}(-1)^{i} \sigma^{-2 i} a_{i v} \int_{-\infty}^{\infty} x^{2(v-i)} f(x) d x=\sum_{i=0}^{v}(-1)^{i} \sigma^{-2 i} a_{i v} \mu_{2(v-i)},
\end{array}
$$

где $a_{i v}$ - коэффициенты при переменной $x$ в полиномах Эрмита.

Коэффициенты $c_{2 s}$ выражаются следующим образом:

$$
\begin{aligned}
& c_{4}=\mu_{4} / \sigma^{4}-3 ; \quad \text { (эксцесс) } \\
& c_{6}=\mu_{6} / \sigma^{6}-15 \mu_{4} / \sigma^{4}+30 ; \\
& c_{8}=\mu_{8} / \sigma^{8}-28 \mu_{6} / \sigma^{6}+210 \mu_{4} / \sigma^{4}-315 ; \\
& c_{10}=\mu_{10} / \sigma^{10}-45 \mu_{8} / \sigma^{8}+630 \mu_{6} / \sigma^{6}-3150 \mu_{4} / \sigma^{4}+3780 ; \\
& c_{12}=\mu_{12} / \sigma^{12}-66 \mu_{10} / \sigma^{10}+1485 \mu_{8} / \sigma^{8}-13860 \mu_{6} / \sigma^{6}+51975 .
\end{aligned}
$$

Выражение (2) для ортогонального разложения плотности $f(x)$ с учетом полученных результатов принимает вид:

$$
f(x)=(2 \pi)^{-1 / 2} \sigma^{-1} \exp \left(-x^{2} / 2 \sigma^{2}\right)\left[1+\sum_{s=2} \frac{c_{2 s}}{(2 s) !} H_{2 s}\left(x / \sigma^{2}\right)\right],
$$

в котором $\sigma^{2}$ и $\mu_{2 s}$ вычисляются по исходной плотности $f(x)$, а выражения для четных полиномов Эрмита имеют следующий вид:

$\mathrm{H}_{4}(y)=y^{4}-6 y^{2}+3$;

$\mathrm{H}_{6}(y)=y^{6}-15 y^{4}+45 y^{2}-15$;

$\mathrm{H}_{8}(y)=y^{8}-28 y^{6}+210 y^{4}-420 y^{2}+105$;

$\mathrm{H}_{10}(y)=y^{10}-45 y^{8}+630 y^{6}-3150 y^{4}+4725 y^{2}-945$;

$\mathrm{H}_{12}(y)=y^{12}-66 y^{10}+1485 y^{8}-13860 y^{6}+51975 y^{4}-62370 y^{2}+10395$.

В приведенных выражениях $y=x / \sigma^{2}$. 
Таким образом, ортогональное разложение плотности распределения вероятностей погрешности навигационных измерений является аналитическим описанием закона их распределения в явном виде.

Выводы и перспектива дальнейшей работы по данному направлению

В работе произведен анализ возможностей применения ортогонального разложения плотностей распределения погрешностей навигационных измерений с помощью полиномов Эрмита. Получены выражения для полиномов Эрмита и коэффициентов разложения для ненормированной плотности нормального закона. В явном виде получены ортогональные разложения плотностей на базе нормированного и ненормированного нормального закона. В дальнейшем целесообразно рассмотреть ортогональные разложения плотностей смешанных законов.

\section{ЛИТЕРАТУРА}

1. Кондрашихин В.Т. Определение места судна / В.Т. Кондрашихин - М.: Транспорт, 1989. - 230с.

2. 1. Ткаченко А.С. Совершенствование методов контроля и прогноза места судна. Автореф. дис. канд. техн. наук: 05.22.13/ ОНМА. - Одесса, 2009. $24 \mathrm{c}$.

3. Астайкин Д.В. Оценка точности координат судна при избыточных измерениях/ Астайкин Д.В., Сикирин В.Е., Ворохобин И.И., Алексейчук Б.M. - Saarbrucken, Deutschland/ Германия: LAP LAMBERT Academic Publishing, 2017. - $274 \mathrm{c}$.

4. Ткаченко А.С. Применение обобщенных пуассоновских распределений для описания навигационных погрешностей / А.С. Ткаченко, В.Г. Алексишин. // Судовождение. - 2008. - № 15. - С. 185 -189.

5. Крамер Г. Математические методы статистики / Г. Крамер - М.: Мир. 1975. - 648 c. 